\title{
Commentary on Canonne (2018): Listening to Improvisation
}

\author{
MANUEL ANGLADA-TORT [1] \\ Audio Communication Group, Technische Universität Berlin
}

\begin{abstract}
The target study explores whether evaluations of the same piece of music differ under two distinct listening conditions: listening to a piece described either as an improvisation or as a preexisting composition. Participants $(N=16)$ in the two conditions listened to the same musical piece and provided verbal evaluative judgements. The author used a grounded theory approach to analyze listeners' responses, reporting different listening experiences in the two groups. The findings provide unique insights to enable a greater understanding of the nature of the aesthetics of improvisation. In this commentary, I first discuss the strengths of the article, followed by methodological considerations and suggestions for future research. I then present a short literature review and discussion of what I consider the most relevant topic in relation to this study, namely, the effects of contextual information on subjective evaluations.
\end{abstract}

Submitted 2018 April 25; accepted 2018 August 5.

KEYWORDS: improvisation, aesthetics, music evaluation, contextual information

Do listeners respond differently to the same musical piece when presented as an improvisation, as opposed to when presented as a live performance of a composition? If so, which are the main perceptual and evaluative aspects that differ? By presenting an identical music recording to highly trained musicians under two different listening conditions, the target paper highlighted numerous key differences in the perception and evaluation of the same piece of music, contributing to the study of the aesthetics of improvised music.

\section{STRENGTHS OF LISTENING TO IMPROVISATION}

The target paper is novel in exploring a topic that, to the best of my knowledge, has not yet been addressed in the scientific literature: how aesthetic responses to a given musical piece depend on whether the piece is presented as an improvisation. As noted by the author, the topic of improvised music has already been discussed by philosophers of music (e.g., Brown, 2000; Day, 2010); however, this topic has not yet been investigated empirically. Thus, the target study makes an important contribution to our limited knowledge of aesthetic responses to improvisation, with implications for other fields, including music perception, music education, performance evaluation, music judgments and preferences, and aesthetics in general.

Another strength of the target article is the methodological approach used, namely, a grounded theory approach of constant comparison (Henwood \& Pidgeon, 2004). Participants' interviews (which lasted up to one hour) were transcribed and coded to establish thematic connections and generate abstract themes to allow the synthesis and organization of the data. This grounded theory approach provides insights that would be unattainable if only using quantitative methods, providing a comprehensive and detailed picture of what happens when people are listening to music under the influence of contextual information (improvisation vs. composition). As far as I know, this is the first study in the published literature that used a grounded theory approach to explore the effects of contextual information on music evaluation.

Overall, the results of the target paper indicate that when experienced as a composition, the same piece was judged more negatively than when presented as an improvisation. However, the use of grounded theory enables a broader understanding of why these evaluations were negative. For instance, a recurrent theme in the composition group was that "the piece was seen as missing any kind of formal organization on a large time scale", which resulted in negative judgments. By contrast, this lack of organization was not 
experienced as a negative aspect in the improvisation group, as trained musicians did not expect improvised music to have a well-defined structure.

The results also suggest that the experiences in the two groups were quite different. Listeners in the composition condition experienced the piece as a "sonic product", paying attention to acoustic effects, imperfections, and the lack of structure. By contrast, listeners in the improvisation group experienced the same piece in a different way. The imperfections and lack of organization were expected and considered a byproduct of the improvisational process, suggesting that listeners were superimposing "ethical" aspects associated with improvisations, rather than evaluating the "quality" of the music itself. Moreover, listeners in the improvisation group did not just perceive "sounds", but also the intentions behind them, identifying the music as a process of communication between musicians.

These findings inspire many topics for discussion and future research. Amongst others, the author pointed out the role of "modal considerations" (i.e., knowing how a given musical piece has been produced). One concept that I found particularly interesting is the relationship between ontology and aesthetics; that is, our perception of music (and aesthetic stimuli in general) seems to rely strongly on the category under which the object in question is being perceived. If true, this raises intriguing questions, for instance: to what extent do we like music simply because it is categorized within our favorite genres or styles? Could we overcome the stigmas associated with classical music amongst young people simply by telling them that the music is not classical?

\section{METHODOLOGICAL CONSIDERATIONS AND SUGGESTIONS}

Grounded theory can be a highly informative and useful approach to explore new research topics. However, this technique also has important limitations, the first being the small number of participants (in the target study there was a total of 16 participants, eight in each group). Although this number is justified by the lengthy work that this approach requires (interviews, transcriptions, and data analysis), I question whether it can be a representative sample of the general population.

The second and third points concern two limitations briefly mentioned by the author: a potential interviewer bias and the limited generalizability of using only one piece of music. The author of the study was the same researcher who conducted the interviews and subsequent analysis, generating the thematic connections and abstract themes. This practice can be susceptible to several biases, including stereotyping, inconsistency in questioning, intuition, and confirmation bias (i.e., the tendency to look for information supporting pre-conceived beliefs).

Using only one piece of music, which was originally an improvisation, can also be an important constraint. Any of the reported results could be due to trained musicians simply identifying and preferring the musical piece in its natural form (i.e., improvisation), or disliking a piece that was presented under the wrong category (i.e., composition). The author attempted to address this issue by conducting a pilot study to select the piece of music that was more ambiguous with regards to its category (i.e., a musical piece that was almost equally perceived as an improvisation and as a preexisting composition). However, to generalize the findings of the target paper, future studies will need to test a wider range of music stimuli, varying not only in category but also in genre and style.

As a general suggestion for overcoming these limitations when designing similar experiments, future research could incorporate both quantitative and qualitative techniques. In this mixed methods approach, a small proportion of the participants could first experience the interview process and provide detailed evaluations. Based on the qualitative analysis of this data, the researcher could then design a quantitative test using a larger number of musical pieces and employing different rating scales to measure the main themes identified in the first phase. This approach would allow researchers to substantially increase the number of participants and music stimuli in a relatively cost-efficient way as well as utilizing inferential statistics to test hypotheses and calculate effect sizes.

Furthermore, it would be interesting to compare the findings from the target article, which used a sample of highly trained musicians, to a group of non-musicians; would non-musicians, compared to musicians, be affected differently by knowing that the musical piece was an improvisation? Perhaps musicians are more susceptible to this information given their extra knowledge, which could generate hypercritical judgements and specific expectations, resulting in negative evaluations when they are not satisfied. 
Finally, when possible, I would recommend the use of a within-participants designs in this type of study, useful for investigating non-musical factors because they allow for the study of their effects on each individual participant while the music remains the same (Anglada-Tort \& Müllensiefen, 2017).

\section{THE IMPACT OF CONTEXTUAL INFORMATION ON SUBJECTIVE EVALUATIONS}

The effects observed in Canonne's study (2018) are a classic example of the influence of contextual information on subjective evaluations.[2] The target paper briefly mentions the relevance of extrinsic factors in listeners' evaluation of music (e.g., Kroger \& Margulis, 2016), but this topic could be addressed in more detail. This section provides an overview of this research area.

In the real world, music is often presented with information of all kinds and forms - e.g., artists' names, song titles, the genre of the music, number of listens, number of likes, price, descriptions of the performer's career, or the composer's intentions. This information likely has an impact on music listening.

When identical recordings are presented with information suggesting that the performer is a professional musician, listeners evaluate the same music stimuli significantly more favorably (e.g., liking, quality, pitch and rhythm accuracy), than when presented with information suggesting a less skilled musician (Anglada-Tort \& Müllensiefen, 2017; Duerksen, 1972; Kroger \& Margulis, 2016). In a functional magnetic resonance imaging (fMRI) study, Aydogan et al. (2018) presented the same music stimuli under two different descriptors about the performer (professionals vs. student), finding that listeners' evaluations were significantly more positive in the professional condition; more importantly, the authors identified different patterns of brain activation in the ventromedial prefrontal cortex.

Extrinsic information presented with music also has a significant impact on emotional responses to music, influencing whether a musical piece is perceived as positive or negative (Margulis, Levine, SimchyFross, \& Kroger, 2017; Vuoskoski \& Eerola, 2013). North and Hargreaves (2005) played songs presented either as 'suicide-inducing' or 'life-affirming'; in both cases, the same pieces of music were perceived just as they were framed. When evaluating music for advertising purposes, industry professionals are also influenced by contextual information, resulting in biased judgements of music aesthetics and the cost associated with the use of the music (Anglada-Tort, Keller, Steffens, \& Müllensiefen, 2018). In fact, the mere manipulation of linguistic properties of names and titles presented with musical pieces has a significant impact on aesthetic and value judgments of music (Anglada-Tort, Steffens, \& Müllensiefen, 2018).

Finally, in the memory domain, the presence of post-event misinformation about a given music piece can impair listeners' ability to remember the music (Anglada-Tort, Baker, \& Müllensiefen, 2018).

The effects of contextual information on subjective judgments have been found in most other domains besides music. Plassmann, O’Doherty, Shiv, \& Rappel (2007) presented the same wine product labeled with different prices (i.e., $\$ 5, \$ 10, \$ 35, \$ 45$, and $\$ 90$ ); the higher the price of the bottle, the higher the pleasure people experienced when drinking the wine. In the olfactory domain, presenting the same smell with two different descriptors, "cheddar cheese" or "body odor", resulted in different judgments of pleasantness, with "body odor" causing a significantly less pleasurable experience (De Araujo, Rolls, Velazco, \& Cayeux, 2005). In the visual domain, when pictures of artworks were either labeled as "gallery" or "computer generated", aesthetic evaluations of the same artworks were significantly higher in the highprestige situation (i.e., the gallery condition; Kirk, Skov, Hulme, Christensen, \& Zeki, 2009). Note that these studies used fMRI techniques and found different patterns of brain activations, identifying the medial prefrontal cortex as an important region involved in the modulation of contextual information.

This growing body of research empirically supports the notion that music perception is contextdependent (e.g., Sloboda, 1999). Nonetheless, it is important to mention that the magnitude of the effects observed in the reviewed literature are generally small. Contrastingly, the target paper suggests that contextual information plays a more important role on music perception and evaluation, with the potential of changing entire listening experiences. These inconsistencies could be due to differences in the experimental methodology or the type of explicit information under study.

Perhaps explicit information effects are outside of our conscious control, aligning with the notion of heuristic processing (Chaiken, 1980; see Kahneman, 2011, for a review). If so, one should consider knowledge from behavioral economics and the psychology of decision making. For instance, the effects of contextual information could be reduced to the heuristic of anchoring (Tversky \& Kahneman, 1974), a decision rule based on the assumption that people rely heavily on the first piece of information offered to "anchor" subsequent judgements and interpretations. Contextual information could therefore be anchoring music evaluations and choices in an unconscious and automatic manner. 
To me, this area of research opens many interesting avenues and raises questions for future studies: Which are the main psychological mechanisms underlying contextual information effects? Are these mechanisms domain-specific? To what extent is music perception determined by contextual information, compared to the other two sources of variability (i.e., the music itself and the listener)? Today, these questions remain unanswered.

\section{CONCLUDING REMARKS}

The target article investigates a new type of contextual information and provides an excellent starting point for anyone interested in the study of the aesthetics of improvisation. It seems relatively easy to manipulate listeners' music experiences by simply changing a piece of descriptive information regarding how the music was produced. However, numerous limitations were identified and future research will need to overcome them to generalize the observed effects, including the use of a larger sample size and a wider range of music stimuli. I would also like to encourage future research to examine similar effects in a sample of non-professional musicians as well as employ within-participants designs. To the best of my knowledge, the target study is the first to use a grounded theory approach to explore contextual information effects on music evaluation. Studies on this subject (as the ones outlined above) normally rely on the use of rating scales that are claimed to measure certain subjective evaluative dimensions, such as music aesthetics. Yet, the experience of reviewing the target paper makes me reconsider the efficiency of quantitative techniques used on its own, inspiring new ways to design future experiments, and adding to the ongoing debate between humanities and sciences.

\section{ACKNOWLEDGMENTS}

This work was supported by a PhD studentship from the "Studienstiftung des Deutschen Volkes" (Bonn, Germany). This article was copyedited by Scott Bannister and layout edited by Kelly Jakubowski.

\section{NOTES}

[1] Correspondence concerning this commentary should be addressed to Manuel Anglada-Tort, Department of Audio Communication, Technische Universität Berlin, Berlin, Germany. E-mail: m.anglada.tort@campus.tu-berlin.de

[2] Contextual information has also been referred in the literature as explicit information, extrinsic information, framing effects, semantic context, extrinsic factors, contextual factors, nonmusical factors, and extramusical factors.

\section{REFERENCES}

Anglada-Tort, M., Baker, T., \& Müllensiefen, D. (2018). False memories in music listening: exploring the misinformation effect and individual difference factors in auditory memory. Memory, 1-16. https://doi.org/10.1080/09658211.2018.1545858

Anglada-Tort, M., Keller, S., Steffens, J., \& Müllensiefen, D. (2018). Consider the source: The effect of source bias on professional assessment of music quality and worth. Paper presented at the 15th International Conference on Music Perception and Cognition combined with the 10th triennial conference of the European Society for the Cognitive Sciences of Music, Graz, Austria.

Anglada-Tort, M., \& Müllensiefen, D. (2017). The repeated recording illusion: The effects of extrinsic and individual difference factors on musical judgements. Music Perception, 35(1), 92-115. https://doi.org/10.1525/mp.2017.35.1.94

Anglada-Tort, M., Steffens, J., \& Müllensiefen, D. (2018, April 5). Names and titles matter: The impact of linguistic fluency and the affect heuristic on aesthetic and value judgements of music. Psychology of Aesthetics, Creativity, and the Arts. Advance online publication. https://doi.org/10.1037/aca0000172 
Aydogan, G., Flaig, N., Ravi, S. N., Large, E. W., McClure, S. M., \& Margulis, E. H. (2018). Overcoming bias: Cognitive control reduces susceptibility to framing effects in evaluating musical performance.

Scientific Reports, 8(1), 6229. https://doi.org/10.1038/s41598-018-24528-3

Brown, L. B. (2000). "Feeling My Way": Jazz improvisation and its vicissitudes. A plea for imperfection. The Journal of Aesthetics and Art Criticism, 58, 113-123. https://doi.org/10.2307/432090

Chaiken, S. (1980). Heuristic versus systematic information processing and the use of source versus message cues in persuasion. Journal of Personality and Social Psychology, 39(5), 752. https://doi.org/10.1037/0022-3514.39.5.752

Day, W. (2010). The ends of improvisation. The Journal of Aesthetics and Art Criticism 68, 291-296.

De Araujo, I. E., Rolls, E. T., Velazco, M. I., Margot, C., \& Cayeux, I. (2005). Cognitive modulation of olfactory processing. Neuron, 46(4), 671-679. https://doi.org/10.1016/j.neuron.2005.04.021

Duerksen, G. L. (1972). Some effects of expectation on evaluation of recorded musical performance. Journal of Research in Music Education, 20(2), 268-272. https://doi.org/10.2307/3344093

Henwood, K., \& Pidgeon, N. (2004). Grounded theory in Psychological Research. In P. M. Camic, J. E. Rhodes, and L. Yardley (Eds.), Qualitative research in psychology: Expanding perspectives in methodology and design (pp. 131-156). Washington, DC: American Psychological Association.

Kahneman, D. (2011). Thinking, fast and slow. New York: Farrar, Straus and Giroux.

Kirk, U., Skov, M., Hulme, O., Christensen, M. S., \& Zeki, S. (2009). Modulation of aesthetic value by semantic context: An fMRI study. Neuroimage, 44(3), 1125-1132. https://doi.org/10.1016/j.neuroimage.2008.10.009

Kroger, C., \& Margulis, E. H. (2016). "But they told me it was professional": Extrinsic factors in the evaluation of musical performance. Psychology of Music, 45(1), 49-64. https://doi.org/10.1177/0305735616642543

Margulis, E. H., Levine, W. H., Simchy-Gross, R., \& Kroger, C. (2017). Expressive intent, ambiguity, and aesthetic experiences of music and poetry. PloS ONE, 12(7), e0179145. https://doi.org/10.1371/journal.pone.0179145

North, A. C., \& Hargreaves, D. J. (2005). Brief report: Labelling effects on the perceived deleterious consequences of pop music listening. Journal of Adolescence, 28(3), 433-440. https://doi.org/10.1016/j.adolescence.2004.09.003

Plassmann, H., O'Doherty, J., Shiv, B., \& Rangel, A. (2008). Marketing actions can modulate neural representations of experienced pleasantness. Proceedings of the National Academy of Sciences, 105(3), 1050-1054. https://doi.org/10.1073/pnas.0706929105

Sloboda, J. A. (1999). Everyday uses of music listening: A preliminary study. In S. W. Yi (Ed.) Music, mind and science (pp. 354-369). Seoul: Western Music Research Institute.

Tversky, A., \& Kahneman, D. (1974). Judgment under uncertainty: Heuristics and biases. Science, 185(4157), 1124-1131. https://doi.org/10.1126/science.185.4157.1124

Vuoskoski, J. K., \& Eerola, T. (2013). Extramusical information contributes to emotions induced by music. Psychology of Music, 43(2), 262-274. https://doi.org/10.1177/0305735613502373 